\title{
Non-adherence with ACE inhibitor treatment is common in heart failure and can be detected by routine serum ACE activity assays
}

\author{
A D Struthers, G Anderson, R J MacFadyen, C Fraser, T M MacDonald
}

\begin{abstract}
Objective-To assess whether serum angiotensin converting enzyme (ACE) activity during routine clinical practice accurately reflects patient adherence to ACE inhibitor treatment for chronic heart failure (CHF). Design-Retrospective assessment of ACE inhibitor adherence and serum ACE activity measurements.

Setting-Teaching hospital outpatient department

Patients and interventions-During 199495, serum ACE was measured in $73 \mathrm{CHF}$ patients who were routinely attending the heart failure clinic at Ninewells Hospital. At the same time, the medicines monitoring unit collected data on whether and when prescriptions for ACE inhibitors were redeemed at community pharmacies, which enabled each patient's adherence over a prolonged period to be assessed.

Main outcome measures-Routine collected serum ACE measurements were correlated with measured adherence with ACE inhibitor treatment.

Results-In total, $18 \%$ of $\mathrm{CHF}$ patients appeared to exhibit $<70 \%$ adherence with their ACE inhibitor treatment with 34\% exhibiting less than $85 \%$ adherence and $58 \%$ exhibiting $<100 \%$ adherence. A serum ACE activity of $>12 \mathrm{u} / 1$ gave $91 \%$ positive predictive accuracy that the patient was $<100 \%$ adherent with their ACE inhibitor treatment. At the other extreme, a serum ACE $<6.5 \mathrm{u} / 1$ gave $81 \%$ positive predictive accuracy that the patient was $>85 \%$ adherent with ACE inhibitor treatment.

Conclusions-Non-adherence with ACE inhibitor treatment was found to be common in patients with CHF. The simple, inexpensive test of serum ACE activity can be used in CHF patients to identify many, although not all, non-adherent patients so that adherence enhancing strategies can be targeted towards them. Further work is clearly required to explore the precise clinical use of this promising test.

(Heart 1999;82:584-588)
\end{abstract}

Keywords: angiotensin converting enzyme inhibitors; heart failure; compliance

Biochemical Medicine, Ninewells Hospital

C Fraser

Correspondence to: Professor Struthers.

Accepted for publication 17 May 1999
Over the last 10 years many large clinical trials have defined the optimal treatment for chronic heart failure (CHF). The most successful treatment by far has been angiotensin converting enzyme (ACE) inhibitors which improve mortality, morbidity, and hospitalisation rates. ${ }^{1}$ In order for individual patients to gain maximum benefit from their ACE inhibitor treatment, it is obviously important that they adhere to this treatment. ${ }^{2}$ Yet many studies suggest that non-adherence with drug treatment is common in CHF patients and has dire consequences. ${ }^{3} \mathrm{CHF}$ patients tend to be elderly and to be on multiple drug treatment which are both factors known to enhance non-adherence. Most previous studies of adherence in CHF are based on self reporting of adherence which is notoriously inaccurate. The best objective study of adherence in CHF is limited to elderly recipients of digoxin through Medicaid where prescription data showed that, on average, elderly CHF patients took none of their prescribed digoxin for 111 days per year, with large interindividual variation. ${ }^{4}$ An important practical problem for the clinician is to identify in clinical practice whether an individual $\mathrm{CHF}$ patient is adherent or not. Without this information, it is impossible to target adherence enhancing strategies to those who require it. $^{2}$ In order to overcome this problem, we recently described the novel idea of using serum ACE activity measurements to assess adherence with ACE inhibitor treatment. ${ }^{5}$ In our preliminary clinical trial, serum ACE activity was an excellent marker of the recent ingestion of an ACE inhibitor drug which discriminated readily between ingesting an ACE inhibitor and ingesting a placebo. ${ }^{5}$ One feature of serum ACE which makes it a very useful measure of adherence is that serum ACE is suppressed equally by different doses of ACE inhibitors, which means that unsuppressed serum ACE levels are caused by poor adherence rather than by the prescribed dose being inadequate. ${ }^{6}$

Our previous results were obtained in a contrived situation where we deliberately altered the subject's adherence. ${ }^{5}$ We now wanted to determine whether this concept could be applied in day to day clinical practice in an unselected and heterogeneous group of $\mathrm{CHF}$ outpatients - that is, could serum ACE activity measurements accurately identify nonadherence in routine, unselected CHF outpatients? This is a crucial step in assessing any new clinical test since clinical trials usually recruit a highly selected group of patients who are often atypical of routine patients. This is even more important when trying to assess adherence as patients who are poorly adherent are particularly unlikely to volunteer for or to be accepted in to most clinical trials. To do this, 


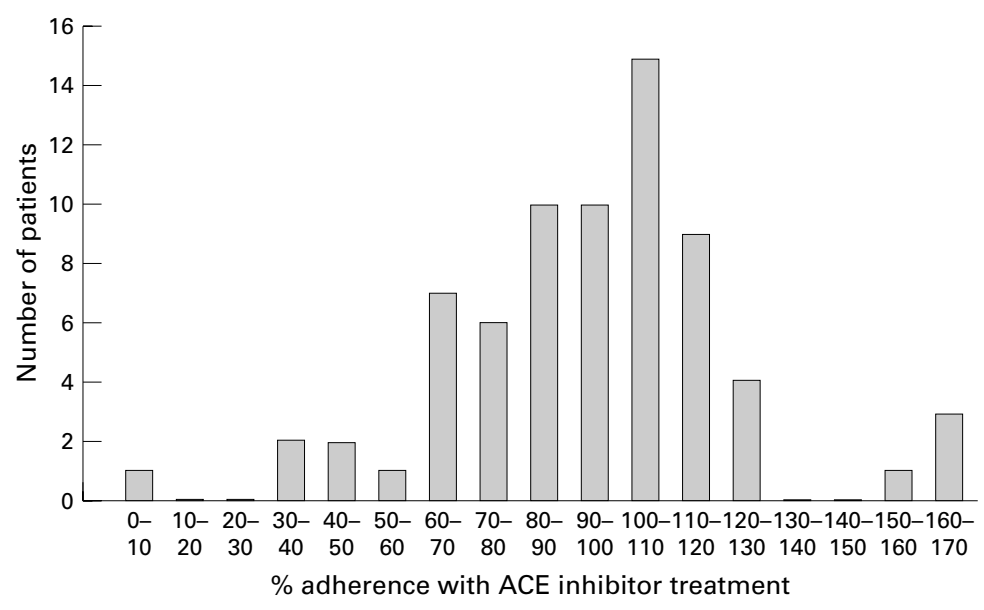

Figure 1 Overall level of adherence with ACE inhibitor treatment in CHF patients (two high ones omitted for clarity). we employed a local computerised system whereby all redeemed drug prescriptions for all routine patients are recorded. This same overall system has been used previously to show that brittle diabetes in adolescents is usually caused by non-adherence with insulin.?

\section{Methods}

This study was carried out using the record linkage of the medicines monitoring unit (MEMO) of the University of Dundee. The database contains information gathered from Tayside community prescriptions for all drugs from January 1993 and details of Tayside hospital admissions from 1980 as collected in Scottish morbidity record 1 . Data are linked by a unique patient identifier, a 10 digit number that comprises date of birth and sex. The methods of collection for this database have been described in further detail elsewhere. ${ }^{8}$

SUBJECTS

The subjects for this study were attending the heart failure clinic at Ninewells Hospital, Dundee, UK during 1995. Once per month, all consecutive patients attending this clinic who were on an ACE inhibitor had venous blood samples taken at between 14:00 and 16:00 $(\mathrm{n}=115)$. Sera were analysed for ACE activity in the directorate of biochemical medicine, Ninewells Hospital. Additional details recorded included each subject's community number, which was essential for record linkage.

\section{EXCLUSION CRITERIA}

People who were prescribed captopril $(n=18)$ were excluded from the study as captopril has a particularly low affinity for serum ACE; captopril dissociates from ACE in ex vivo serum, hence making serum ACE an unreliable measure of the in vivo situation and also of adherence with captopril. People who lived in north east Fife $(n=14)$ were excluded from the study as MEMO does not record prescribing data from outwith Tayside. It was not possible to calculate accurately drug adherence in people who cashed less than three prescriptions over the study period $(n=7)$ and in people with ambiguous drug regimens $(n=3)$-for example, patients instructed by their family physician to take drug as required. These two groups were therefore also excluded. A total of 73 patients were included in the study.

CALCULATING ACE INHIBITOR ADHERENCE OVER THE STUDY PERIOD

The ACE inhibitor prescriptions dispensed to each patient between January 1993 and September 1995 were abstracted from the MEMO database. The number of days when medication was available to each individual was taken as the numerator. The denominator was the total length of time the patient was in the study and was determined by subtracting the date that the first prescription was issued in the study period from the date of the last day when treatment was available from the last prescription in the study period. The total number of days in hospital (if any) during the study period was subtracted from the denominator. The numerator was divided by the denominator to express adherence as a percentage for each patient.

SERUM ACE ACTIVITIES

The serum ACE activity for each individual patient was measured between 1995 and 1997. Over half the study group $(60 \%)$ had more than one ACE assay in this period and in these cases the mean ACE activity was taken for each person.

ACE activity was assayed by monitoring change in absorbance at $340 \mathrm{~nm}$ of the hydrolysis of furylacrylolylphenylalanylglycylglycine (FAPGG) to FAP and GG (Sigma-Aldrich Chemical Company, Poole, Dorset, UK) on a Roche MIRA analyser (Roche Diagnostic Systems, Welwyn Garden City, Herts, UK).

CALCULATING DIURETIC DRUG DOSE

The average daily dose of diuretic drug over the study period was calculated for each person in the study. This was used as an approximation for the severity of their heart failure.

\section{Results}

\section{DEMOGRAPHICS}

Almost a quarter $(23 \%)$ of the group were female, with ages ranging from 52-83 years (mean 71 years). The age range of men was wider, from 41-87 years (mean 67 years). Of the ACE inhibitors prescribed, $95 \%$ of patients took either lisinopril or enalapril in roughly equal proportions $(56 \% v 44 \%)$. The aetiology of heart failure was ischaemic heart disease in $90 \%$ of patients, with a mean duration of symptoms of 22 months.

MEAN ACE INHIBITOR ADHERENCE

Figure 1 shows the distribution of ACE inhibitor adherence: $18 \%$ of individuals had $<70 \%$ adherence, with $34 \%$ having $<85 \%$ adherence, and $58 \%$ having $<100 \%$ adherence.

MEAN ACE INHIBITOR ADHERENCE STRATIFIED BY SERUM ACE ACTIVITIES

Figure 2 shows the relation between serum ACE activity and ACE inhibitor adherence. 


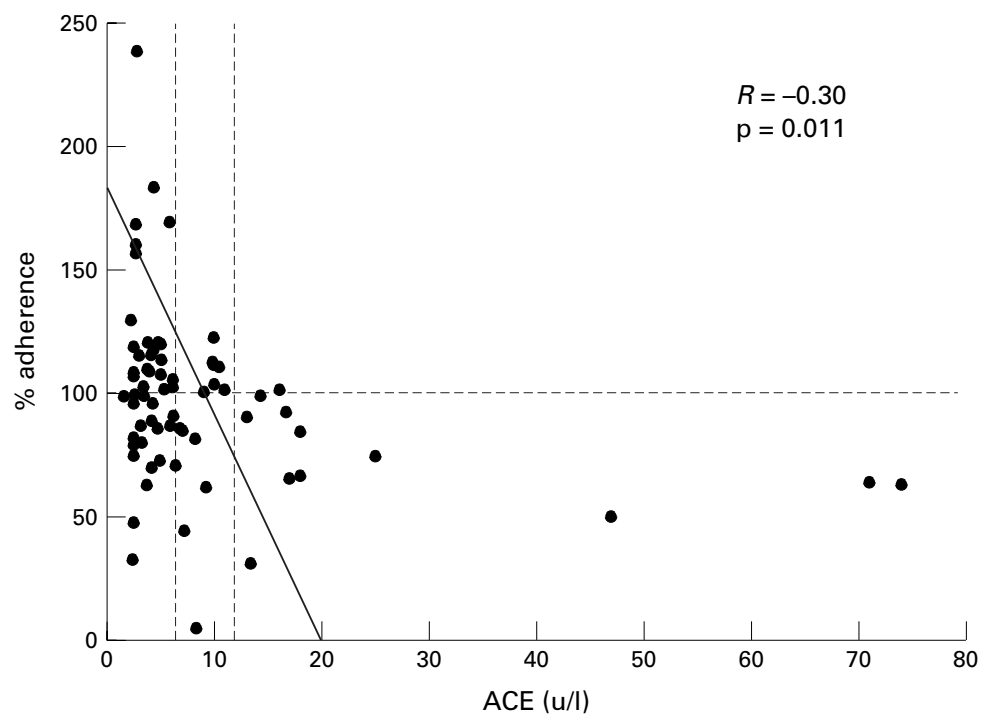

Figure 2 Relation between serum ACE activity measurements and adherence with $A C E$ inhibitor treatment in CHF patients. Linear correlation $r=-0.30, p=0.011$, although the relation is really curvilinear.
Table 2 Mean ACE inhibitor adherence stratified by dose of $A C E$ inhibitor

\begin{tabular}{lllc}
$\begin{array}{l}\text { Dose/day ACE } \\
\text { inhibitor (mg) }\end{array}$ & $\begin{array}{l}\text { Number of } \\
\text { patients }\end{array}$ & $\begin{array}{l}\text { Mean serum } \\
\text { ACE (u/l) }\end{array}$ & $\begin{array}{l}\text { Mean ACE inhibitor } \\
\text { adherence (\%) }\end{array}$ \\
\hline $1-5$ & 13 & 8.5 & 96 \\
$6-10$ & 31 & 9.7 & 100 \\
$11-20$ & 23 & 8.4 & 99 \\
$20+$ & 7 & 7.1 & 80 \\
\hline
\end{tabular}

MEAN ACE INHIBITOR ADHERENCE STRATIFIED BY DOSE OF ACE INHIBITOR

The study group was stratified according to the daily dose of ACE inhibitor (table 2). There was no significant difference between the mean ACE activities in the subgroups with taking different amounts of drug. There was a difference in mean ACE inhibitor adherence between those people taking doses $<20 \mathrm{mg}$ per day and those taking doses $>20 \mathrm{mg}$ per day, but this finding is not reliable since there were only seven people in the group taking $>20 \mathrm{mg}$ per day.

MEAN ACE INHIBITOR ADHERENCE STRATIFIED BY DOSE OF DIURETIC DRUG

The study population was divided into two groups: those taking $<50 \mathrm{mg}$ of a diuretic per day $(n=31)$; and those taking $\geqslant 50 \mathrm{mg}$ of a diuretic per day $(n=42)$. The diuretic doses were taken as approximate measures of disease severity. The mean ACE inhibitor adherence for these groups was $98 \%$ in those on $<50 \mathrm{mg}$ per day frusemide equivalent and $96 \%$ in those on $\geqslant 50 \mathrm{mg}$ per day frusemide equivalents ( $\mathrm{p}=0.8$, no significant difference).

\section{Discussion}

Adherence to treatment in the real world is notoriously difficult to assess and it is inevitable that all assessments of adherence will be less than perfect. In this study, we have probably used the two best possible measures of adherence-that is, redeemed prescriptions and a biochemical measure of the drug in vivo. It is difficult to imagine how these two measures of adherence will ever be improved upon unless one was to install secret cameras in patients' homes. Added confidence in our two measures is given in this study by two facts. Firstly, these two independent measures generally agreed with each other. Secondly and most importantly of all, neither technique alerted the patient that their adherence was being measured and hence their normal drug taking behaviour was not perturbed by the techniques designed to measure their adherence.

In this study, we were therefore studying unselected patients who were behaving as they normally do. This ideal situation is unique and seldom occurs in most clinical research. Other techniques for assessing adherence have serious limitations making them much less valuable. For example, even if a researcher was physically present to record whether a patient swallowed their tablets or not, this would alert the patient that their adherence was being measured and they would then behave atypically. Another technique is a device which measures when a pill bottle is opened but they suffer from many problems-for example, they $81 \%$ PPV of $>85 \%$ adherence $50 \%$ have $<85 \%$ adherence $50 \%$ have $>85 \%$ adherence $75 \%$ PPV of $<85 \%$ adherence $91 \%$ PPV of $<100 \%$ adherence

PPV, positive predictive value. 
alert the patient to behave more adherently, they make opening pill bottles difficult for arthritic hands, and when they suffer from technological failure, one does not know whether apparent non-adherence is true or is caused by intermittent technological failure. In addition, patients often open pill bottles to check whether they have taken their tablets and this leads to odd results. One might imagine that plasma digoxin concentrations would be useful in assessing adherence but the interindividual pharmacokinetic variability with digoxin is so large that no one has seriously attempted using them for this purpose.

It is the use of ACE inhibitor in CHF which affords the unique opportunity of assessing drug adherence by measuring serum ACE activity. Serum ACE is already measured routinely in most hospital laboratories as a way of assessing sarcoidosis. In our hospital serum ACE activity is assayed on an automated system. This makes its routine use cheap and simple, which cannot be said for other proposed ways of measuring ACE inhibitor adherence biochemically. ${ }^{10}$ Fortunately, all ACE inhibitors act via the final common mechanism of inhibiting ACE, which gives us this unique opportunity. No other class of drugs has such a common final pathway which is so easily measured.

Our main finding is that a serum ACE activity $>12 \mathrm{u} / 1$ has high positive predictive value at identifying poor adherence with ACE inhibitor treatment in CHF. One could argue that a high positive predictive value is more important than a high negative predictive value here because the subject of non-adherence should only be raised with an individual patient if they are indeed non-adherent. Otherwise, the suggestion that a patient might be non-adherent could lead to a deterioration in the doctor-patient relationship. This does, however, mean that some non-adherent patients will be missed by serum ACE, but this is still an improvement on current clinical practice where all non-adherent patients are missed. The accurate identification of non-adherence in an individual patient should lead to the targeted introduction of adherence enhancing strategies. ${ }^{1112}$

The apparently non-adherent individuals with suppressed serum ACE activities are probably demonstrating the "toothbrush" effect - that is, when going to the dentist, most individuals will brush their teeth even if they did not do so often beforehand. It is a limitation of serum ACE that it responds to the recent ingestion of ACE inhibitor rather than to its long term ingestion. In that sense, serum ACE is more akin to blood sugar for assessing diabetes rather than to a $\mathrm{HbA}_{\mathrm{IC}}$ measurement. Nevertheless, it will be of enormous clinical benefit to be able to identify a large proportion of non-adherent patients, especially when the test used will do so with very few false positives.

The prescribing data in our study also allows us to ascertain adherence levels in general in routine $\mathrm{CHF}$ outpatients. This has only previously been done with digoxin treatment in elderly Medicaid patients, the latter obviously being a highly selected group. In this study of routine unselected outpatients in the UK, $18 \%$ of patients had $<70 \%$ adherence with their ACE inhibitor treatment and 34\% $<85 \%$ adherence. In our study, ACE inhibitor nonadherence was not related to disease severity.

It is now being realised that plasma angiotensin II (AII) does not remain suppressed in all patients during chronic ACE inhibitor treatment. It is also now realised that patients with AII reactivation, despite ACE inhibitor treatment, have a worse prognosis. ${ }^{13}{ }^{14}$ Various explanations have been proposed for this AII reactivation such as non-ACE pathways generating AII, or excess angiotensin I building up and so producing AII despite ACE inhibition. Another explanation might be non-adherence with the ACE inhibitor treatment. This is a crucial issue to resolve since non-adherence would best be tackled by adherence enhancing strategies, whereas this will have no impact on patients with non-ACE pathways or AI build up where changing to an AII receptor antagonist would be a more appropriate response. Clearly, different patients may well have different reasons for their AII reactivation but serum ACE assays could be used in practice to see whether non-adherence is a possible explanation in each individual. In uncertain cases, the measurement of serum ACE activity after the supervised administration of an ACE inhibitor is a further option to confirm whether the patient is non-adherent.

The unique feature of our study is that it provides "proof of the concept" that routine serum ACE measurements can reflect ACE inhibitor adherence in routine clinical practice. Our results apply specifically to blood being taken 5-8 hours after enalapril or lisinopril but clearly there are several issues of detail which need to be clarified by future studies. Whether the dose of the ACE inhibitor influences serum ACE is one such issue although, at peak drug effect, the relation between dose and serum ACE is known to be flat, which is why serum ACE was only $1.4 \mathrm{u} / 1$ less with quadrupling the dose of ACE inhibitor in this study. This agrees with our previous finding when serum ACE fell by $3 \mathrm{u} / 1$ when the dose of lisinopril was quadrupled from $5 \mathrm{mg}$ per day to $20 \mathrm{mg}$ per day. ${ }^{15}$ It also agrees with van Veldhuisen and colleagues who found that serum ACE was suppressed equally by three very different ACE inhibitor doses. ${ }^{6}$ This flat dose-response curve at peak drug effect is probably because small doses usually lower serum ACE down to near its limits of biochemical detection.

Another possible factor is an individual's ACE genotype. As in normal clinical practice, we did not have information on our patients' ACE genotype. However, in the presence of an ACE inhibitor, serum ACE is only $3.6 \mathrm{u} / 1$ lower in subjects with the II genotype as compared to the DD genotype. ${ }^{15}$ Therefore, the ACE inhibitor dose and the ACE genotype will probably at most each alter serum ACE by 3-4 $\mathrm{u} / 1$, which will have little impact on the clinical utility of this test, except in borderline cases with serum ACE around $12 \mathrm{u} / 1$. We need also to ensure that serum ACE concentrations 
do not change as the disease progresses, although the frusemide data in this paper and our other data suggest that this is not the case.

Serum ACE only measures the serum activity of this one enzyme; it is not altered by diuretics, salt or disease progression which are known to activate renin and angiotensin II but not to alter serum ACE per se. In order to use this test outwith the 5-8 hour postdose time studied here, we would need more information on the time course of serum ACE as our data only apply to the common situation of blood samples taken 5-8 hours after oral ingestion. Current data with lisinopril suggests that $10 \mathrm{mg}$ lisinopril reduces serum ACE by $93 \%$ at 6 hours, $80 \%$ at 24 hours, $60 \%$ at 48 hours, and $40 \%$ at 72 hours. ${ }^{6}{ }^{16}$ This suggests that it might not matter when in the first 24 hours the blood sample is taken because serum ACE is fairly stable over that period.

One limitation of our study is that we were assessing adherence by whether patients had redeemed prescriptions and hence whether they had tablets available at home to ingest. Clearly, if they had tablets available to ingest for only $60 \%$ of the time, then their maximum possible adherence is $60 \%$; it could be less if they did not swallow all the available tablets but it is almost impossible for it to be greater. In other words, we did not assess the number of tablets actually swallowed; rather we assessed each individual's maximum possible adherence rates. This is presumably why some patients appeared to have $>100 \%$ adherence-that is, they cashed new prescriptions before the old prescription was finished. This is more likely to be because of misplacing the old supply of tablets than overdosing themselves. It also means that our figure of $34 \%$ of patients taking $<85 \%$ of their ACE inhibitor treatment is an optimistic figure-that is, it could be that the number of patients who are non-adherent is even higher than $34 \%$ but it is virtually impossible for the figure to be less than $34 \%$. Despite this limitation in our methodology, our way of assessing adherence is a notable improvement on all other ways of assessing adherence.

In summary, we have developed and shown that serum ACE activity assays are of some value in routine clinical practice as a measure of adherence with ACE inhibitor treatment. This simple test is clearly not perfect but it can certainly be used to identify many nonadherers. It should also help determine whether AII reactivation in individual patients is caused by non-adherence or by some other mechanism since the appropriate response is different in each case. Lastly, our study provides a means to help assess adherence with ACE inhibitors in research studies. Trials which do not rigorously assess adherence could be giving us false negative information because non-adherers dilute out any clinical benefit. One possible example of this is the second cooperative new Scandinavian enalapril survival study (CONSENSUS II) ${ }^{17}$ which may have given a different result from all other postmyocardial infarction studies of ACE inhibitors because of adherence differences between these trials.

1 Studies of Left Ventricular Dysfunction Investigators. Effect of enalapril on survival in patients with reduced left ventricular ejection fractions and congestive heart failure. N Engl f Med 1991;325:293-302.

2 Haynes RB, McKibbon KA, Kanani R. Systematic review of randomised trials of interventions to assist patients to follow prescriptions for medications. Lancet 1996;348:3836.

3 Ghali JK, Kadakis S, Cooper R, et al. Precipitating factors eading to decompensation of heart failure. Arch Intern Med 1988; 148:2013-6.

4 Monane M, Bohn RL, Gurwitz JH, et al. Non compliance with congestive heart failure therapy in the elderly. Arch Intern Med 1994;154:433-7.

5 MacFadyen RJ, Struthers AD. Practical assessment of compliance with ACE inhibitor therapy: a novel approach. $\mathcal{F}$ Cardiovasc Pharmacol 1997;29:119-24.

6 van Veldhuisen DJ, Genth-Zotz S, Braouwer J, et al. High versus low dose ACE inhibition in CHF. 7 Am Coll Cardiol 1998;32:1811-18.

7 Morris AD, Boyle DIR, McMahon AD, et al for DARTS/ MEMO collaboration. Adherence to insulin treatment, glycaemic control, and ketoacidosis in insulin dependent glycaemic control, and ketoacidosis in insulin

8 Evans JMM, McDevitt DG, MacDonald TM. The Tayside medicines monitoring unit (MEMO): a record-linkage system for pharmacovigilance. Pharmaceutical Medicine 1995; 9:177-84.

9 Nussberger J, Brunner D, Keller I, et al. Measurement of converting enzyme activity by antibody tapping of generated angiotensin II: comparison with two other methods. Am 7 Hypertens 1992;5:393-8.

10 Azizi M, Ezan E, Nicolet L, et al. High plasma level of N-acetyl-seryl-aspartyl-lysyl-proline: a new marker of chronic ACE inhibitor. Hypertension 1997;30:1015-19.

11 Corlett AJ. Aids to compliance with medication. BMF 1996; 313:926-9.

12 Rich MW, Beckham V, Wittenberg C. A multidisciplinary intervention to prevent the readmission of elderly patient with CHF. N Engl F Med 1995;333:1190-5.

13 Pouleur H, Konstan MA, Benedict CR, et al. Progression of LV dysfunction during enalapril therapy: relationship with neurohormonal reactivation. Circulation 1993;88(4 part 2):I-293.

14 Swedberg K, Eneroth P, Kjekshus J, et al for the CONSENSUS Trial Study Group. Hormones regulating cardiovascular function in patients with severe $\mathrm{CHF}$ and their relation to mortality. Circulation 1990;82:1730-5.

15 Davidson NC, Coutie WJ, Webb DJ, et al. Hormonal and renal differences between low dose and high dose ACEI treatment in patients with chronic heart failure. Heart 1996;75:576-81.

16 Biollaz J, Schelling JL, Jacot-Des-Combes B, et al. Enalapril maleate and a lysine analogue (MK 521) in normal volunteers: relationship between plasma drug levels and the renin angiotensin system. Br f Clin Pharmacol 1982;14: 363-8.

17 Swedberg K, Held P, Kjekshus J, et al. Effects of the early administration of enalapril on mortality in patients with acute myocardial infarction: results of the cooperative new Scandinavian enalapril survival study II (CONSENSUS II). N Engl f Med 1992;327:678-84. 